\title{
Resistance and aerobic training in the treatment of type 2 diabetes mellitus
}

\begin{abstract}
Diabetes Mellitus is considered a chronic disease of epidemic proportions that affects approximately 415 million adults. Several studies reported the benefits of exercise training for this syndrome treatment. The aim of this study was to summarize the major findings of aerobic, resistance, and combined training influence in patients with Type 2 Diabetes Mellitus. The studies showed that different modalities of exercise exert positive effects on insulin sensitivity, exercise capacity, glycemic control, vascular function, and other risk factors associated with Type 2 Diabetes Mellitus. As a conclusion, physical exercise is a safe and effective strategy to prevent and mitigate the changes associated with Type 2 Diabetes Mellitus, besides it is possible to get benefits with continuous aerobic (low, moderate or high intensity), interval, combined or resistance training.
\end{abstract}

Keywords: type 2 diabetes mellitus, exercise, aerobic exercise, resistance training, highintensity interval training, glycemic control, cardiovascular disease
Volume 4 Issue 5 - 2017

\author{
Bruna Marmett,' Ramiro Barcos Nunes ${ }^{2,3}$ \\ 'Laboratory of Atmospheric Pollution, Federal University of \\ Health Sciences of Porto Alegre (UFCSPA), SA \\ 2Laboratory of Experimental Physiology, Federal University of \\ Health Sciences of Porto Alegre (UFCSPA), SA \\ ${ }^{3}$ Research Department- Sul-Rio-Grandense Federal Institute of \\ Education, Science and Technology, SA
}

Correspondence: : Ramiro Barcos Nunes, Federal University of Health Sciences of Porto Alegre, 245 Sarmento Leite Street, Zip code 90050 I70, Porto Alegre, RS, SA, Tel 555 I 3303 8800, Email ramiro.barcos@gmail.com

Received:September 14, 2017 | Published: October 16, 2017
Abbreviations: 1RM, 1-repetition maximum; AE, aerobic training; ATP, adenosine triphosphate; CAT, catalase; $\mathrm{CB}$, combined training; DM, diabetes mellitus; DM2, type 2 diabetes mellitus; FLI, fatty liver index; FMD, flow-mediated dilatation; GLUT4, glucose transporter type 4; HbA1C, glycated hemoglobin; HDL-c, high density lipoprotein cholesterol; HI, high intensity; HOMA-IR, homeostatic model assessment of insulin resistance; $\mathrm{HR}_{\mathrm{MAX}}$, maximum heart rate; HRR, heart rate reserve; IL-6, interleukin 6; INT, interval training; LDL-c, low density lipoprotein cholesterol; LW, low intensity; NNOS, neuronal nitric oxide synthase; OGTT, oral glucose tolerance test; RT, resistance training; REHIT, reduced-exertion high-intensity interval training; SOD, superoxide dismutase; TNF- $\alpha$, tumor necrosis factor alpha; TSH, thyroid-stimulating hormone; VAI, visceral adiposity index; $\mathrm{VO}_{2 \mathrm{MAX}}$, maximum oxygen consumption; $\mathrm{VO}_{2 \mathrm{PEAK}}$, peak oxygen consumption

\section{Introduction}

Diabetes mellitus (DM) is reported as a non-transmissible chronic disease of epidemic proportions and considered one of the largest health emergencies of the 21 st century. ${ }^{1} \mathrm{DM}$ is characterized by the inability of the pancreas to adequately secrete insulin or by tissue resistance to this hormone action. ${ }^{2}$ The World Health Organization estimated that DM was responsible for 1.2 million deaths worldwide. ${ }^{3}$ Elucidating this fact, the International Diabetes Federation (2015) recently announced that approximately 415 million adults suffer from DM and associated complications. ${ }^{1}$ These numbers negatively surpassed the 366million forecast for 2030, in an estimation made in the last decade. ${ }^{4}$ This data indicates the exponential prevalence of this disease, causing a strong impact on public health. In the ranking of countries/number of people with diabetes, China occupies the first position with 109.6million, followed by India, the United States and

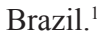

Among different pathophysiology of this disease, Type 2 DM (DM2), also known as non-dependent insulin, usually affects middleaged individuals who present altered levels of insulin resistance, as well as deficiencies in this hormone secretion in response to the destruction of $\beta$-pancreatic cells. ${ }^{2}$ In this scenario, less glucose will be captured and used by muscle tissue, causing metabolic and vascular changes. ${ }^{5}$ emphasizes that hyperglycemia is the key to a series of biochemical events that elevates oxidative stress, causing oxidation of LDL molecules, activation of coagulation processes, as well as stimulating the adhesion of leukocytes and platelets, which contribute to thrombus formation, leading a predisposed environment to endothelial dysfunction development. ${ }^{5}$ In consequence, DM has been associated with the development of other cardiovascular complications. ${ }^{2,6}$

The discovery of glucose transport independent of insulin's action and its modulation by muscle contraction led the exercise to become a promising therapy for DM2 treatment. Resistance and aerobic training (AE) compose the two main axes of treatment, which may provide different or even similar results by opposite pathways. ${ }^{2}$ Thus, a synthesis of the main findings involving these modalities in DM2 patients is necessary. Considering the introduced above, we aimed to review the current literature on the effect of resistance training (RT) and endurance on glycemic control, vascular function, and other risk factors associated with DM2. Main outcomes from selected articles are listed in Table 1.

Table I Main outcomes from exercise intervention in DM2

\begin{tabular}{lll}
\hline Author/Year & Intervention & Main outcome \\
\hline & & $\uparrow$ VO2PEAK, HDL-c \\
Kadoglou et al. ${ }^{7} \quad$ Low-intensity aerobic training for 12 weeks $(n=54)$ & $\downarrow$ HOMA-IR, HbAIC \\
& & $\downarrow$ LDL-c, total cholesterol, triglycerides \\
& $\downarrow$ Systolic blood pressure
\end{tabular}


Table continued..

\begin{tabular}{|c|c|c|}
\hline Author/Year & Intervention & Main outcome \\
\hline Krause et al. ${ }^{9}$ & $\begin{array}{l}\text { Low }(30-40 \% \text { VO2MAX) and moderate }(55-65 \% \\
\text { VO2MAX) intensities of endurance training for } \\
\text { l } 6 \text { weeks }(n=25)\end{array}$ & $\begin{array}{l}\leftrightarrow \text { VO2MAX } \\
\leftrightarrow \text { HbAIC, HOMA-IR } \\
\leftrightarrow \text { IL-6,TNF- } \alpha, \text { HDL-c, LDL-c } \\
\downarrow \text { Protein carbonyls } \\
\uparrow \text { Catalase, NNOS }\end{array}$ \\
\hline Silva et al. ${ }^{8}$ & $\begin{array}{l}\text { Low (LW } 50-60 \% \text { HRMAX) and high }(75-85 \% \text { of } \\
\text { HRMAX) intensity training for 6weeks }(n=31)\end{array}$ & $\uparrow$ Functional capacity and endothelium-dependent dilation of $\mathrm{HI}$ group \\
\hline Balducci et al. ${ }^{10}$ & $\begin{array}{l}\text { Low and high intensity of AE ( } 55 \% \text { or } 70 \% \text { of } \\
\text { predicted maximal oxygen consumption), and RT } \\
\text { ( } 60 \% \text { or } 80 \% \text { of predicted I-RM) for } 12 \text { months } \\
(n=303)\end{array}$ & $\begin{array}{l}\uparrow \text { VO2MAX, IRM, HDL-c } \\
\downarrow \text { HOMA-IR, HbAIC } \\
\downarrow \text { Systolic and diastolic blood pressure }\end{array}$ \\
\hline Mitranun et al. ${ }^{\prime \prime}$ & INT and continuous training for 12 weeks $(n=43)$ & $\begin{array}{l}\uparrow \text { VO2MAX, IRM, vasodilatation capacity } \\
\downarrow \text { HOMA-IR, LDL-c } \\
\uparrow \text { Endothelial function markers, antioxidant activity, oxidative stress in INT } \\
\text { training }\end{array}$ \\
\hline Ruffino et al. ${ }^{12}$ & $\begin{array}{l}\text { REHIT and moderate-intensity walking for } 8 \text { weeks } \\
(n=16)\end{array}$ & $\begin{array}{l}\uparrow \text { Aerobic fitness } \\
\downarrow \text { Arterial pressure } \\
\leftrightarrow \text { OGTT, blood lipid profile, body composition }\end{array}$ \\
\hline Francois et al. ${ }^{13}$ & $\begin{array}{l}\text { Acute session of cardio and resistance INT } \\
\text { training }(n=35)\end{array}$ & $\begin{array}{l}\uparrow \text { Endothelial function I and } 2 \mathrm{~h} \text { after RT INT } \\
\uparrow \text { Endothelial function I } \mathrm{h} \text { after cardio INT }\end{array}$ \\
\hline $\begin{array}{l}\text { Geirsdottir et } \\
\text { al. }{ }^{14}\end{array}$ & Resistance training for 12 weeks $(n=237)$ & $\begin{array}{l}\uparrow \text { Muscle strength, palmar pressure } \\
\leftrightarrow \mathrm{HbAIC} \text {, lean mass, triglycerides }\end{array}$ \\
\hline Mavros et al. ${ }^{15}$ & $\begin{array}{l}\text { High-intensity progressive and low-intensity of RT } \\
\text { for } 12 \text { months }(n=103)\end{array}$ & $\begin{array}{l}\leftrightarrow \text { Body composition } \\
\downarrow \text { Adipose mass } \\
\uparrow \text { Insulin sensitivity of responders to high-intensity RT }\end{array}$ \\
\hline Church et al. ${ }^{16}$ & $A E, R T$, and $C B$ training for 9months $(n=262)$ & $\begin{array}{l}\downarrow \mathrm{HbAIC} \text { in } \mathrm{CB} \\
\uparrow \mathrm{VO} 2 \mathrm{PEAK} \text {, muscular strength } \\
\uparrow \text { Lean mass in RT }\end{array}$ \\
\hline Sparks et al. ${ }^{17}$ & $\begin{array}{l}\text { Muscle biopsies from participants performing AE, } \\
\text { RT, and CB training for 9months }(n=262)\end{array}$ & $\begin{array}{l}\uparrow \text { Mitochondrial DNA copies, citrate synthase in RT and CB } \\
\uparrow \text { Oxidation of medium chain fatty acids in } A E \text { and } C B \\
\downarrow \text { Mitochondrial dysfunction }\end{array}$ \\
\hline Oliveira et al. ${ }^{12}$ & $A E, R T$, and $C B$ training for 12 weeks $(n=48)$ & $\begin{array}{l}\leftrightarrow \mathrm{HbAIC} \text {, lipid profile, blood pressure, liver function } \\
\leftrightarrow \text { Anthropometric measures } \\
\leftrightarrow \text { Cortisol and TSH } \\
\uparrow \text { VO2PEAK, SOD and CAT activity, concentration of nitrate in AE }\end{array}$ \\
\hline Taylor et al. ${ }^{19}$ & $\begin{array}{l}\text { AE and RT in moderate ( } 30 \% \text { to } 45 \% \text { of HRR and } \\
75 \% \text { of the } 8 \text { RM) or high-intensity training }(50 \% \\
\text { to } 65 \% \text { of the HRR and } 100 \% \text { of the } 8 \text { RM) for } \\
\text { 3months }(n=21)\end{array}$ & $\begin{array}{l}\uparrow \text { Muscle strength } \\
\uparrow \text { Exercise capacity } \\
\uparrow \text { Physical function }\end{array}$ \\
\hline $\begin{array}{l}\text { Yalamanchi et } \\
\text { al. }^{20}\end{array}$ & AE and RT training for 6 months $(n=50)$ & $\begin{array}{l}\downarrow \text { Fasting glucose } \\
\downarrow \text { Fat mass Total } \\
\uparrow \text { Lean body mass }\end{array}$ \\
\hline Balducci et al. ${ }^{21}$ & $\begin{array}{l}\text { AE and RT training with structured counseling and } \\
\text { counseling alone for } 12 \text { months }(n=606)\end{array}$ & $\begin{array}{l}\downarrow \text { Fatty liver index and visceral adiposity index in counseling group } \\
\leftrightarrow \text { Liver enzymes }\end{array}$ \\
\hline
\end{tabular}

I-RM, I- repetition maximum; AE, aerobic training; CAT, catalase; CB, combined training; DM2, Type 2 diabetes mellitus; HbAIC, glycated hemoglobin; HI, high intensity; HDL-c, high density lipoproteins cholesterol; HOMA-IR, homeostatic model assessment of insulin resistance; HR ${ }_{\text {MAX }}$, maximum heart rate; HRR, heart rate reserve; IL-6, interleukin 6; INT, interval training; LDL-c, low density lipoprotein cholesterol; NNOS, neuronal nitric oxide synthase; OGTT, oral glucose tolerance test; RT, resistance training; SOD, superoxide dismutase; TNF- $\alpha$, tumor necrosis factor alpha; TSH, thyroid-stimulating hormone; VO ${ }_{2 M A x}$, maximum oxygen consumption; $\mathrm{VO}_{2 \mathrm{PEAK}}$, peak of oxygen consumption 


\section{Discussion}

Several studies reported the beneficial effects of physical exercise as an intervention on pathophysiological processes associated to DM2, supporting the guidelines recommendation of exercise prescription to this disease treatment. ${ }^{6-8}$ The first studies conducted with this purpose chose aerobic training as an intervention, possibly due to the relationship with the cardiovascular system and the ease of control of the intervening variables. Therefore, ${ }^{7}$ randomized 54 adults to two groups, one remained untreated and the other was assigned to 12 weeks of low-intensity aerobic training. At the end of the study, it was observed that the AE group had improved peak oxygen consumption $\left(\mathrm{VO}_{2 \mathrm{PEAK}}\right)$, homeostatic model assessment of insulin resistance (HOMA-IR), glycated hemoglobin (HbA1C), and lipid profile (HDL-c, LDL-c, total cholesterol, and triglycerides), as well as reduced systolic blood pressure. These effects were not observed in the control group, evidence that, even with low-intensity exercises, it is possible to attenuate the metabolic alterations related with DM2.

In this regard, ${ }^{9}$ evaluated 25 patients with DM2 performing endurance training in different intensities, moderate $\left(55-65 \% \mathrm{VO}_{2 \mathrm{MAX}}\right)$ and low $\left(30-40 \% \mathrm{VO}_{2 \mathrm{MAX}}\right)$. The authors investigated the impact of these interventions on the inflammatory profile, oxidative stress, and nitric oxide production in obese subjects with or without DM2. At the end of the intervention, there were no changes in the main variables analyzed $\left(\mathrm{VO}_{2 \mathrm{MAX}}, \mathrm{HbA1C}\right.$, HOMA-IR, IL-6, TNF- $\alpha$, HDL-c, LDL-c). The exceptions were protein carbonyls analysis, a marker of oxidative stress, which reduced in obese individuals with DM2, and the activity of the antioxidant enzyme catalase (CAT), which increased after the exercise intervention of moderate intensity. Additionally, the same group increased NNOS enzyme expression, evidencing a higher production of nitric oxide. Although the experimental design is the strength of the study, characterized by a well-defined prescription and intensity control of exercise, the reduced sample size represented its main limitation. However, it is plausible to conclude that endurance training can positively modulate the action of the CAT by reducing oxidative damage in obese subjects with DM2, especially if performed at moderate intensity. ${ }^{9}$

Contributing to this issue, ${ }^{8}$ compared high-intensity training (HI $75-85 \%$ of $\mathrm{HR}_{\mathrm{MAX}}$ ) to low-intensity training ( $\mathrm{LW} 50-60 \% \mathrm{HR}_{\mathrm{MAX}}$ ) in 31 middle-aged diabetic patients. After the randomization process and six weeks of intervention, the authors observed that individuals in the HI group were active for a longer time on the maximal exercise test than LW group. ${ }^{8}$ Regarding endothelial function, there was an expressive improvement in endothelium-dependent dilation in the HI group compared to LW group. Based on these data, authors concluded that the HI training is effective in improving the functional capacity and the endothelium-dependent vasodilator response in obese patients with DM2, being an important preventive strategy. Similar result was observed in the Italian Diabetes and Exercise Study, 303 patients (HI and LW intensities groups) improved exercise capacity $\left(\mathrm{VO}_{2 \mathrm{MAX}}\right)$, insulin sensitivity (HOMA-IR), glycemic control (HbA1C), lipid profile (HDL-c) and strength (1RM), besides reducing systolic and diastolic blood pressure and becoming more physical active after 12 months of treatment. ${ }^{10}$ Among the aforementioned variables, the $\mathrm{HI}$ and LW groups differed only in muscle strength, HbA1C, and total cholesterol in favor of the HI group. It is important to highlight the concomitant performance of strength training $(60 \% 1 \mathrm{RM})$ with endurance protocols, which may have influenced the outcome of the research. These studies show that both training intensities are beneficial in DM2 treatment. ${ }^{8,10}$
Another commonly prescribed methodology for improving the cardiovascular system is the interval or intermittent training (INT), which is characterized by periods of high intensity mixed with recovery periods. Elucidating this practice effect, ${ }^{11}$ compared INT method to continuous training performed for 12 weeks in 43 individuals with DM2. This study identified that both techniques provided improved exercise capacity $\left(\mathrm{VO}_{2 \mathrm{MAX}}\right)$, insulin sensitivity (HOMA-IR), lower members strength (1RM), vasodilatation capacity (\% flow-mediated dilatation (\% FMD) of brachial artery), and LDL-c levels. However, only participants submitted to the INT training had additional $\mathrm{VO}_{2 \mathrm{MAX}}$ and vascular reactivity gains, as well as a positive response in glycemic control (HbA1C), lipid profile (HDL-c), endothelial function markers, antioxidant activity, and oxidative stress. Thus, the authors concluded that both methodologies are beneficial in the prevention and treatment of DM2. However, the INT training seems to provide additional gains compared to the traditional method. ${ }^{11}$

Likewise, ${ }^{12}$ investigate INT modality compared to AE training in 16 men with DM2. Participants completed a reduced-exertion highintensity interval training (REHIT) and moderate-intensity walking for 8 weeks with a 2 months wash-out period between interventions. As a result, both modalities decreased resting mean arterial pressure and improve aerobic fitness, REHIT being five-fold higher in improving aerobic fitness than moderate intensity walking. However, neither intervention significantly improved OGTT, glycemic control measured using continuous glucose monitors, blood lipid profile or body composition. Indicating that REHIT led to a higher aerobic fitness than walking, however, neither strategies were effective interventions for improving insulin sensitivity or glycemic control in DM2 patients in a short-term period. ${ }^{12}$ While, ${ }^{13}$ examined the effect of a single session of cardio and resistance INT training in 35 participants with DM2 and control groups, which was composed of highly trained and untrained normoglycemic adults. The methodology of exercise consists of seven $1 \mathrm{~min}$ cycling at $85 \%$ of peak power with $1 \mathrm{~min}$ recovery for cardio INT, and the same pattern of seven $1 \mathrm{~min}$ intervals using leg resistance exercises for RT INT. Endothelial function, assessed with a \% FMD of the brachial artery, performed at baseline, $1 \mathrm{~h}$, and $2 \mathrm{~h}$ after exercise, was improved in all RT INT groups. However, DM2 participants demonstrated most robust results in every analyses moment. After cardio INT, a \%FMD improvement in DM2 was observed at $1 \mathrm{~h}$ post-exercise compared with control. Authors concluded that even an acute session of RT training improves endothelial function throughout the $2 \mathrm{~h}$ post-exercise period in DM2 patients, indicating that the long-term impact of resistance exercise performed in an interval pattern is warranted. ${ }^{13}$

Based on the previous studies, it is possible to perceive the beneficial action of aerobic exercise in DM2 treatment. According to Colberg SR, et al., ${ }^{2}$ the byproduct of energy phosphorylation, called CAMP, is recognized as a mediator responsible for the insulinindependent activation of glucose transporter proteins (GLUT4). ${ }^{2}$ Since their concentration is closely linked to adenosine triphosphate (ATP) depletion, it is perfectly acceptable that strength training or resistive training could achieve results similar or superior to those observed previously. In this respect, ${ }^{14}$ evaluated resistance training for 12 weeks on body composition, muscle strength, and glycemic control in 237 elderly randomized to three groups: healthy, pre-diabetic, and DM2. At the end of the intervention, all participants had gains in muscle strength of lower members and in palmar pressure, which may have reflected in the higher distance walked in the walk test. So, all groups improved performance in the functional tests. However, the 
gain of lean mass expected with the training was only obtained in healthy and pre-diabetic groups. Regarding physiological variables, any group showed a reduction in $\mathrm{HbA1C}$ concentrations. The serum levels of glucose and triglycerides were lower in healthy subjects only. Thus, ${ }^{14}$ concluded that elderly DM2 patients may have similar benefit from RT training than healthy individuals, based on functional performance and muscle strength. However, changes in $\mathrm{HbA1C}$ levels were not detected in this study, possibly because this marker reflects glycemic control over the last 120days. So, it takes a longer period of intervention to assess training influence on this marker. ${ }^{6,14}$

An investigation of ${ }^{15}$ showed RT training influence on insulin resistance and glycemic control in 103 elderly participants with DM2 for 12 months. Patients were randomized to 2 groups: HI progressive RT and LW RT. Authors classified patients in responsive and nonresponsive to RT, representing those who have gained and lost muscle mass, respectively. At the end of the study, it was not observed a difference in the body composition and both groups showed a similar reduction of adipose mass. However, when analyzing the changes in the HOMA-IR and HbA1C indices, adjusted in a responsive and non-responsive subgroup, the authors showed that elderly individuals responsive to HI progressive RT had better insulin sensitivity when paired with nonresponsive elderly in the same group and LW RT group. Regarding $\mathrm{HbA1C}$, the $\mathrm{HI}$ progressive $\mathrm{RT}$ responders had greater reductions than the LW RT responsive and non-responsive HI progressive RT groups. Considering the above, the researchers reported that RT training is effective in improving the glycemic control and insulin sensitivity in elderly patients with DM2. ${ }^{15}$

If considering the outcomes of the studies previously discussed, it is reasonable to deduce that the combination of resistance and aerobic methodologies may provide additional results compared to those methods practiced alone. In this perspective, ${ }^{16}$ compared the effect of aerobic, resistance, and combined (CB) training on HbA1C levels in DM2 patients submitted to 9months of intervention. The authors randomized 262 middle-aged individuals to three training groups (AE, RT and $\mathrm{CB}$ ) and a control group. After training protocols, only the $\mathrm{CB}$ group participants significantly reduced $\mathrm{HbA} 1 \mathrm{C}$ levels when compared to the control group. In relation to the aerobic capacity, the groups $\mathrm{AE}$ and $\mathrm{CB}$ demonstrated values superior to the control. The $\mathrm{VO}_{2 \text { PEAK }}$ of the CB group was higher than the RT group. As expected, there was an increase in the muscular strength of the groups submitted to $\mathrm{CB}$ and $\mathrm{RT}$ training when compared to control and AE groups, with an advantage of RT training over $\mathrm{CB}$ group. All groups reduced adipose tissue and $\mathrm{CB}$ training proved to be more effective in this outcome than the AE training. Only the RT group obtained a lean mass gain at the end of the program. An interesting data not explored by the authors show that in the first two months all intervention groups (AE, RT and $\mathrm{CB}$ ) presented a decrease in HbA1C levels (data not analyzed), however, these values stabilized and returned to baseline levels after the third or fourth month of therapy, perhaps the intensity adjustment may have influenced this response. As the main finding, the authors suggest that $\mathrm{CB}$ training may be more effective in reducing $\mathrm{HbA1C}$ levels when compared to modalities performed alone. ${ }^{16}$

In addition, ${ }^{17}$ performed biopsies in the Vastus lateralis muscle of the 262 patients from the aforementioned study aiming to investigate the influence of AE, RT, and $\mathrm{CB}$ modalities on the mitochondrial density and enzymatic activity in the peripheral musculature. After 9 months of intervention, the authors observed an increase in the number of mitochondrial DNA copies in RT and CB groups, as well as a higher activity of the citrate synthase enzyme, which is vital for the proper functioning of the oxidative pathway. Therefore, the results showed higher mitochondrial density and enzymatic activity in the peripheral musculature, suggesting a better adaptation of oxidative metabolism in the long-term exercise. Another analysis performed by Sparks LM, et al., ${ }^{17}$ showed that the ability of the muscle to oxidize long-chain fatty acids increased in the $\mathrm{AE}, \mathrm{RT}$ and $\mathrm{CB}$ groups. However, only groups $\mathrm{AE}$ and $\mathrm{CB}$ improved the oxidation of medium chain fatty acids. In addition, all groups improved the ability to metabolize pyruvate and acetate, essential substrates in glycolytic metabolism, indicating that mitochondrial dysfunction related to insulin resistance may be ameliorated by different modalities (AE, RT, and CB).${ }^{17}$ According to findings of the Health Benefits of Aerobic and Resistance Training in Individuals with Type 2 Diabetes trial, the CB therapy may be more effective in the treatment of patients with DM2. ${ }^{16,17}$

Recently, ${ }^{12}$ expanded the analyses by investigating the effect of 12 weeks of AE, RT, and CB training on glycemic control, lipid profile, and oxidative stress. Thus, the authors allocated 48 participants in the following groups: AE, RT, $\mathrm{CB}$ and control. Contrary to the initial expectations, no changes were detected in most of the variables investigated (HbA1C, lipid profile, anthropometric, blood pressure, liver function, serum levels of cortisol, and TSH). The authors attributed these results to the reduced sample size and to the excessive caution of subjects on evaluation protocols performance, in order to avoid getting injured, which could have influenced the performance of recommended intensities, even using established criteria of exercise evaluation and prescription (electrocardiogram test, lactate threshold, and 1RM test). In contrast, participants in the AE group improved $\mathrm{VO}_{2 \text { PEAK }}$ levels, SOD and CAT activities. Regarding the higher concentration of nitrate in this group, the data suggested that the $\mathrm{AE}$ training may improve the antioxidant action and increase the bioavailability of nitric oxide, a potent vasodilator, minimizing the deleterious effects of oxidative stress. So, ${ }^{18}$ indicated that this modality may offer additional protection to patients with DM2 due to its antioxidant action. ${ }^{18} \mathrm{~A}$ differential of this work in relation to those published by Church TS et al., ${ }^{16}$ and Sparks et al., ${ }^{17}$ is the shorter period of intervention, which could be insufficient to detect more expressive results, besides the sample selected have a lower body mass index. ${ }^{16-18}$

Also, ${ }^{19}$ investigated the effects of different intensities of AE and RT training for 3months in 21 individuals with DM2. Participants were randomly allocated to receive moderate-intensity training, which consists of $75 \%$ of the $8 \mathrm{RM}$ for RT and $30 \%$ to $45 \%$ of the heart rate reserve (HRR) for AE, or high-intensity training, representing $100 \%$ of the $8 \mathrm{RM}$ for RT and $50 \%$ to $65 \%$ of the HRR for AE. As a result, participants in both groups demonstrated significant and similar gains in muscle strength, exercise capacity, and physical function. Indicating that moderate and high intensity of exercise training may lead to improvements in physical fitness and physical function in people with DM2. However, ${ }^{19}$ highlighted some limitations, as sample size and subjectivity on physical function measurement. ${ }^{19}$ In a contribution to this findings,,$^{20}$ performed supervised aerobic and resistance training intervention for 6months in 50 individuals with DM2. After AE and RT interventions, participants presented total fat mass decreased and total lean body mass increased in association of fasting glucose reduction. So, these data demonstrate that muscle gain and fat loss after ET are positively related to baseline hyperglycemia. ${ }^{20}$

Investigating the same modalities, ${ }^{21}$ evaluated levels of liver enzymes, fatty liver index (FLI), and visceral adiposity index (VAI) in 606 sedentary patients with DM2. Participants were assigned to 
supervised aerobic and resistance training plus structured exercise counseling or counseling alone for 12 months. The counseling group was further randomized to low-to-moderate or moderate-to-high intensity training of equal energy expenditure. As a result, enzymes levels did not change, whereas FLI and VAI decreased significantly in counseling group, but not in counseling alone participants. So, physical activity volume was an independent predictor of both FLI and VAI reductions and a decrease in these variables with supervised training occurs in a volume-dependent manner. ${ }^{21}$

\section{Conclusion}

Based on the information discussed previously, it becomes evident that physical exercise is a safe and efficient strategy, which offers different training options, to prevent and attenuate the pathophysiological changes associated with DM2. It is possible to obtain benefits through aerobic exercises of low, moderate, and high intensity, besides intermittent, combined, and resistance exercise. It is the professional involved that must recognize and prescribe, individually, the best conduct based on clinical, physiological, psychological, and social conditions of each patient. However, only instructions to follow strictly a certain conduct may not be enough, professionals should offer different possibilities of exercise training so the patient could, according to his preferences, have a greater commitment to the treatment. Finally, it is important to emphasize that a good professional must be updated and use all available information for a better guidance.

\section{Acknowledgements}

The authors would like to thank Federal University of Health Sciences of Porto Alegre (UFCSPA), Coordination of Improvement of Higher Education Personnel (CAPES) and Foundation for Research Support of the State of Rio Grande do Sul (FAPERGS).

\section{Conflict of interest}

The authors declare that they have no financial interest or conflict of interest.

\section{References}

1. International Diabetes Federation. IDF Diabetes Atlas update poster. 7th ed. International Diabetes Federation. Brussels, Belgium; 2015.

2. Colberg SR, Sigal RJ, Fernhall B, et al. Exercise and type 2 diabetes: the American College of Sports Medicine and the American Diabetes Association: joint position statement. Diabetes Care. 2010;33(12):147-167.

3. WHO. Global report on diabetes. Geneva: World Health Organization. 2016.

4. Wild S, Roglic G, Green A, et al. Global prevalence of diabetes: estimates for the year 2000 and projections for 2030. Diabetes Care. 2004;27(5):1047-1053.

5. Wajchenberg B. Disfunção Endotelial no Diabetes do Tipo 2. Arq Bras Endocrinol Metab. 2002;46(5):514-519.

6. Sociedade Brasileira De Diabetes. Diretrizes da Sociedade Brasileira de Diabetes 2013-2014. AC Farmacêutica, São Paulo; 2005.
7. Kadoglou NP, Vrabas IS, Kapelouzou A, et al. The impact of aerobic exercise training on novel adipokines, apelin and ghrelin, in patients with type 2 diabetes. Med Sci Monit. 2012;18(5):290-295.

8. da Silva CA, Ribeiro JP, Canto JC, et al. High-intensity aerobic training improves endothelium-dependent vasodilation in patients with metabolic syndrome and type 2 diabetes mellitus. Diabetes Res Clin Pract. 2012;95(2):237-245.

9. Krause M, Rodrigues-Krause J, O’Hagan C, et al. The effects of aerobic exercise training at two different intensities in obesity and type 2 diabetes: implications for oxidative stress, low-grade inflammation and nitric oxide production. Eur J Appl Physiol. 2014;114(2):251-260.

10. Balducci S, Zanuso S, Cardelli P, et al. Effect of high- versus low-intensity supervised aerobic and resistance training on modifiable cardiovascular risk factors in type 2 diabetes; the Italian Diabetes and Exercise Study (IDES). PLoS One. 2012;7(11):492-497.

11. Mitranun W, Deerochanawong C, Tanaka H, et al. Continuous vs interval training on glycemic control and macro- and microvascular reactivity in type 2 diabetic patients. Scand J Med Sci Sports. 2014;24(2):69-76.

12. Ruffino JS, Songsorn P, Haggett M, et al. A comparison of the health benefits of reduced-exertion high-intensity interval training (REHIT) and moderate-intensity walking in type 2 diabetes patients. Appl Physiol Nutr Metab. 2017;42(2):202-208.

13. Francois ME, Durrer C, Pistawka KJ, et al. Resistance-based interval exercise acutely improves endothelial function in type 2 diabetes. $\mathrm{Am} \mathrm{J}$ Physiol Heart Circ Physiol. 2016;311(5):1258-1267.

14. Geirsdottir OG, Arnarson A, Briem K, et al. Effect of 12 week resistance exercise program on body composition, muscle strength, physical function, and glucose metabolism in healthy, insulin-resistant, and diabetic elderly Icelanders. J Gerontol A Biol Sci Med Sci. 2012;67(11):1259-1265.

15. Mavros Y, Kay S, Anderberg KA, et al. Changes in insulin resistance and $\mathrm{HbA} 1 \mathrm{c}$ are related to exercise-mediated changes in body composition in older adults with type 2 diabetes: interim outcomes from the GREAT2DO trial. Diabetes Care. 2013;36(8):2372-2379.

16. Church TS, Blair SN, Cocreham S, et al. Effects of aerobic and resistance training on hemoglobin A1c levels in patients with type 2 diabetes: a randomized controlled trial. JAMA. 2010;304(20):2253-2262.

17. Sparks LM, Johannsen NM, Church TS, et al. Nine months of combined training improves ex vivo skeletal muscle metabolism in individuals with type 2 diabetes. J Clin Endocrinol Metab. 2013;98(4):1694-1702.

18. de Oliveira VN, Bessa A, Jorge ML, et al. The effect of different training programs on antioxidant status, oxidative stress, and metabolic control in type 2 diabetes. Appl Physiol Nutr Metab. 2012;37(2):334-344.

19. Taylor JD, Fletcher JP, Mathis RA, et al. Effects of moderate- versus high-intensity exercise training on physical fitness and physical function in people with type 2 diabetes: a randomized clinical trial. Phys Ther. 2014;94(12):1720-1730.

20. Yalamanchi SV, Stewart KJ, Ji N, et al. The relationship of fasting hyperglycemia to changes in fat and muscle mass after exercise training in type 2 diabetes. Diabetes Res Clin Pract. 2016;122:154-161.

21. Balducci S, Cardelli P, Pugliese L, et al. Volume-dependent effect of supervised exercise training on fatty liver and visceral adiposity index in subjects with type 2 diabetes The Italian Diabetes Exercise Study (IDES). Diabetes Res Clin Pract. 2015;109(2):355-363. 